\title{
Comparison of iPACK and periarticular block with adductor block alone after total knee arthroplasty: a randomized clinical trial
}

\author{
Tayfun Et ${ }^{1,2}\left(\right.$ D $\cdot$ Muhammet Korkusuz $^{2} \cdot$ Betül Basaran $^{1} \cdot$ Rafet Yarımoğlu $^{2} \cdot$ Hatice Toprak $^{1} \cdot$ Ayşegül Bilge $^{1}$. \\ Nuh Kumru² ${ }^{2}$ Ilker Dedeli ${ }^{3}$
}

Received: 4 November 2021 / Accepted: 1 February 2022 / Published online: 14 February 2022

(c) The Author(s) under exclusive licence to Japanese Society of Anesthesiologists 2022

\begin{abstract}
Purpose The infiltration between the popliteal artery and the capsule of the posterior knee (iPACK) has been described to provide analgesia without loss of muscle strength and is effective in functional recovery. This study compared iPACK + ACB (adductor canal block) with PAI (periarticular infiltration) + ACB and ACB alone in terms of postoperative analgesia and functional improvement.

Methods This double-blinded randomized controlled trial included 105 patients undergoing unilateral total knee arthroplasty. Patients received ACB, iPACK + ACB, and PAI + ACB along with spinal anesthesia. The primary outcome was the area under the curve (AUC) numeric rating scale (NRS) at $48 \mathrm{~h}$. Secondary outcomes were cumulative postoperative analgesic consumption within $48 \mathrm{~h}$, timed up-and-go test, range of motion, length of hospital stay, patient satisfaction, and adverse events. Results The 48-h AUC movement NRS score in the iPACK + ACB group was significantly lower than in the PAI + ACB and ACB groups $(p<0.05)$. At the postoperative 48th $\mathrm{h}$, the opioid consumption of the iPACK + ACB group was lower than those of the ACB and PAI + ACB groups $(p<0.001)$. The patients in the iPACK + ACB group had significantly shorter discharge and mobilization days than the ACB and PAI + ACB groups $(p<0.001)$.

Conclusions The adding of an iPACK block to the ACB improves postoperative analgesia and reduces opioid consumption. In addition, this approach improves functional performance and reduces hospital stay.
\end{abstract}

Keywords Total knee arthroplasty $\cdot$ iPACK $\cdot$ Adductor canal block $\cdot$ Periarticular block

\section{Introduction}

Total knee arthroplasty (TKA) is one of the commonly performed major orthopedic surgeries in which most patients experience severe pain in the postoperative period. Optimal postoperative knee analgesia is important for not only patient comfort and satisfaction, but also for accelerating mobilization, functional recovery, and hospital discharge. Multimodal analgesia and motor-protective blocks are

Tayfun Et

drtayfunet@gmail.com

1 Department of Anesthesiology and Intensive Care Medicine, Faculty of Medicine, Karamanoglu Mehmetbey University, Karaman, Turkey

2 Department of Anesthesiology and Intensive Care Medicine, Karaman Training and Research Hospital, Karaman, Turkey

3 Department of Orthopedics and Traumatology, Karaman Training and Research Hospital, Karaman, Turkey increasingly used to facilitate early ambulation and provide superior performance [1].

Adductor canal block (ACB) is popular in patients undergoing total knee arthroplasty owing to its postoperative opioid sparing and motor-protective effects. It is considered as an element of the multimodal analgesia regimens [2]. The PAI (periarticular infiltration) technique is a simple blind technique applied intraoperatively by orthopedic surgeons, and it is based on a systematic infiltration method applied to all knee joint structures, usually by combining local anesthetic and various drug selections. It may have motor-protective effects but may not provide complete analgesia [3]. The sensory coverage of the ACB is limited to the anteromedial part of the knee [4]. TKA patients who received ACB alone as postoperative analgesia may still complain of posterior knee pain [5].

There is increasing interest in local anesthetic infiltration in the space between the popliteal artery and posterior capsule of the knee, which is called iPACK [6]. This approach 
blocks the terminal branches of the genicular nerves and popliteal plexus, which innervate the posterior capsule of the knee joint while sparing the major trunks of the tibial and common peroneal nerves [7]. Accordingly, the iPACK block seems to provide a promising ultrasound-guided motorprotective posterior knee analgesia with a lower probability of nerve or vascular injury [8]. The ACB combined with an iPACK block yields significantly better postoperative numeric rating scale (NRS) scores, knee range of motion, and ambulation distances compared to ACB alone $[9,10]$.

There is a scarcity of studies investigating the combined use of the ACB along with iPACK and PAI. Provision of effective analgesia after TKA can be achieved by blocking the terminal branches of the nerves that innervate the anterior and posterior parts of the knee. The motor-sparing effect created by the blockade of the terminal nerve branches allows early rehabilitation in the postoperative period and reduces incidence of falls in the ward [10]. For effective analgesia, the nerves innervating the posterior part of the knee must be blocked. The iPACK region is considered to involve the nerve network innervating the posterior part of the knee [11]. Hence, nerves innervating the anterior and posterior parts of the knee can be blocked by the combined application of ACB and PACK under ultrasound guidance. This combination also decreases postoperative ambulatory pain scores and increases the compliance of patients to rehabilitation [11].

The primary aim of this prospective, randomized study was to compare the analgesia levels (48th-h area under the curve versus time NRS value) of patients who underwent combined iPACK + ACB block with patients who underwent ACB only and ACB + PAI over the same period. The secondary aim was to evaluate the effect of iPACK and PAI with added ACB on postoperative analgesic consumption and functional recovery.

\section{Materials and methods}

\section{Study design and patient enrollment}

This prospective, single-center, double-blind randomized controlled trial was approved by the Karamanoğlu Mehmetbey University the Ethics Committee of Faculty of Medicine (02-2021/04), and written informed consent was obtained from all participants of the study. The study was registered on Clinicaltrials.gov (NCT04931966; principal investigator: TE; registration date: June 22, 2021) prior to initiation of patient enrollment. From June to August 2021, patients listed to undergo elective, unilateral primary TKA were screened for inclusion.

The participants were randomly divided into three parallel groups using six blocks according to a computerized randomization list: the ACB-only group (ACB); iPACK combined with ACB group (iPACK + ACB); Group undergoing PAI combined with ACB (PAI+ ACB). The anesthesia technician and operating room nurses were not blinded to the status of the patients. None of the anesthetists participating in the study followed the patient in the postoperative period and collected data for research; this was only done by a blinded research assistant. In the postoperative period, the post-anesthesia care unit (PACU) and ward nurses were also blinded to the group distribution. Groups were not unblinded until data collection was completed.

Patients between the ages of 18 and 85 who would have unilateral knee arthroplasty and willing to participate were included in the study. Since we are the only center in the region that performs elective surgery due to COVID-19, the study was completed in a shorter time than anticipated.

Inclusion criteria:

- American Society of Anesthesiologists (ASA) I-III

Exclusion criteria:

- Previous surgery on the same knee

- Liver or kidney failure

- Under 18 and over 85 years old

- Patients undergoing general anesthesia

- Allergy or intolerance to one of the study drugs

- Body mass index (BMI) $>40 \mathrm{~kg} / \mathrm{m}^{2}$

- Chronic use of gabapentin/pregabalin (regular use for more than 3 months)

- Chronic opioid use (opioid use for more than 3 months or daily oral morphine use equivalent of $>5 \mathrm{mg}$ /day for 1 month)

Demographic data, preoperative pain score, and functional performance indicators: The time up go (TUG) test and range of motion (ROM) were recorded 1 day before the surgery by the research assistant in the surgical wards. All patients were instructed how to use the NRS for the assessment of pain intensity.

\section{Multimodal analgesia}

Oral acetaminophen (1000 mg) and oral diclofenac sodium (75 mg tablet) were given to all patients in the ward $30 \mathrm{~min}$ before the surgery.

\section{Preoperative}

The patients were monitored in a standard way. All groups were administered $0.03 \mathrm{mg} / \mathrm{kg}$ midazolam iv, $40 \mathrm{mg}$ esomeprazole iv, dexamethasone $10 \mathrm{mg}$ iv, and prophylaxis against nausea and vomiting. 


\section{Intraoperative}

Spinal anesthesia was performed using $15 \mathrm{mg} 0.5 \%$ hyperbaric bupivacaine in the sitting position at L3-4 or L4-5 intervertebral space. Intraoperative fluid administration and sedation with intravenous propofol were performed by the anesthetist, who was blind to the study.

We applied the sham procedure to the patients. Sham procedure, marking the injection site with a stump needlenon-penetrating into the skin. After spinal anesthesia and sedation were applied to the patients, adductor canal block was applied. Then, after the ultrasound was placed on the popliteal region, the stump was touched with a needle from the outside of the thigh. At the end of the operation, the injection site was covered with a bandage.

\section{Adductor canal block (group ACB) ( $n$ : 35)}

After placing the patients in the supine position, the linear probe of ultrasound (13-6 MHz) was moved from cephalad to caudal and the superficial femoral artery was visualized in the short axis, medial to the vastus medialis, lateral to the sartorius muscle, and anterior to the adductor magnus muscle. A 22 Gauge $\times 100 \mathrm{~mm}$ needle (Braun ${ }^{\circledR}$ Stimuplex) was guided from lateral to medial to this area called the adductor canal using in-plane technique. $20 \mathrm{~mL}$ of $0.5 \%$ bupivacaine was injected to with peri-arterial spread after negative aspiration under sterile conditions.

\section{Adductor + iPACK group (group iPACK + ACB) $(n: 35)$}

$20 \mathrm{~mL}$ of $0.25 \%$ bupivacaine was administered into the adductor channel. After the knee was flexed, the convex probe $(3.5-6 \mathrm{MHz})$ was placed in the popliteal region, and the popliteal artery was identified in the short axis. The ultrasound probe was moved distally from the division of the common peroneal and tibial nerves. The probe was slowly moved towards the popliteal crease until the tibial nerve was defined superficially to the popliteal vessels. The ultrasound probe was moved the level of the junction of the femoral condyles with the femoral shaft. Popliteal artery, tibial and peroneal nerves were visualized and the space between the femur and popliteal artery was determined (Fig. 1). 22 Gauge $\times 100 \mathrm{~mm}$ needle (Braun ${ }^{\circledR}$ Stimuplex) was advanced to this area called iPACK using the lateral to medial in-plane technique. After negative aspiration, $20 \mathrm{ml}$ of $0.25 \%$ bupivacaine was injected and slowly withdrawn until the end of the lateral femoral condyle under sterile conditions [12].

\section{Adductor + PAI (group PAI + ACB) (n: 35)}

$20 \mathrm{~mL}$ of $0.25 \%$ bupivacaine was administered into the adductor canal. TKA was performed by an orthopedic

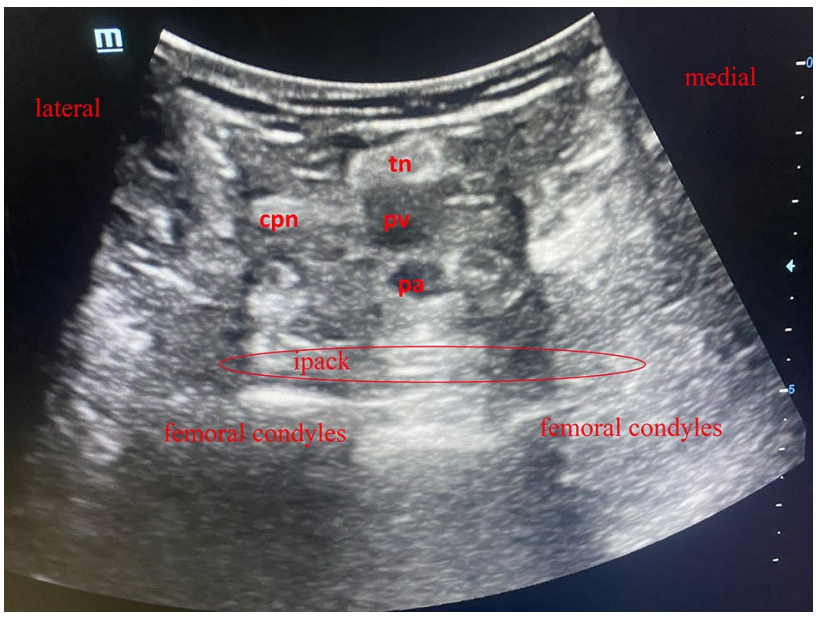

Fig. 1 The iPACK is ultrasound image with a low-frequency curvilinear probe placed along the posterior. tn tibial nerve, peroneal nerve, $p v$ popliteal vein, $p a$ popliteal artery, ipack the interspace between the popliteal artery and capsule of the posterior knee

surgeon by placing three-compartment prostheses with a minimally invasive mini-midvastus approach and using hand-mixed cementing techniques. A thigh tourniquet was applied by all surgeons throughout the operation. $60 \mathrm{ml}$ of local anesthetic cocktail ( $40 \mathrm{ml}$ of $0.25 \%$ bupivacaine $+8 \mathrm{ml}$ $8 \mathrm{mg}$ of morphine $+12 \mathrm{ml}$ of saline) prepared by the nurse were applied by the surgeon to the periarticular region (posterior capsule, collateral ligament, quadriceps muscle) at the end of the operation.

\section{Postoperative}

Multimodal analgesia regimen was used at the postoperative period. The patients were administered a combination of acetaminophen ( $1 \mathrm{~g}$ IV every $6 \mathrm{~h}, 4$ doses), diclofenac (50 mg every $8 \mathrm{~h}$ PO 3 doses, $25 \mathrm{mg}$ for $\geq 75$ years of age), and tramadol $100 \mathrm{mg}$ IV as a rescue analgesia when patient complained of pain with NRS $>4$.

\section{Outcome measures}

- Primary outcome: Analgesic efficiency of the anesthetic modalities in groups; area under the curve for NRS at 48th h was evaluated.

- Secondary outcome: When the first need for analgesic rescue (NRS score $>4$ [0: no pain 10: very severe pain]) was received at $2,4,6,8,12,24$, and $48 \mathrm{~h}$ and the total amount of analgesic were determined.

- The patients were evaluated in terms of Bromage score and incidence of peroneal motor weakness (dorsiflexion of the ankle and toes).

- Length of hospital stay (defined as the days from the end of surgery until discharge). 
o Functional discharge criteria were applied (ability to get dressed independently, ability to sit and rise from a chair/toilet, independence in personal care, mobilization with walker/crutches, and ability to walk $>70 \mathrm{~m}$ with crutches). In addition, adequate pain management with oral drugs was required for discharge.

- Patient satisfaction was assessed at $48 \mathrm{~h}$ postoperatively $(0=$ very dissatisfied, $2=$ somewhat dissatisfied, $3=$ somewhat satisfied, $4=$ very satisfied).

- The patient's first mobilization time was recorded in hours.

- The TUG test (measured in seconds), which required the patient to get up from a chair, walk $3 \mathrm{~m}$, turn, walk back to the chair, and sit, was performed on the 1 st and 2 nd postoperative days

- Postoperative 1st and 2nd-day ROM measurements were recorded.

- Nausea and vomiting scores were recorded (none/middle/ vomiting).

\section{Statistical analysis}

Repeated measures analysis of variance (ANOVA) was used to compare changes in NRS scores between the three groups. Differences in NRS scores in each time point and area under the NRS curve scores were analyzed. Changes in NRS scores within each group were confirmed for $48 \mathrm{~h}$, and categorical variables were analyzed for independence by chi-square test. A priori power analysis was carried out in the GPower 3.1 program to determine the sample size. As a result of the analysis made with a pilot data of 15 people, sample size for a power of 0.80 was calculated as 99 in the three-group sample, with a 5\% margin of error, $95 \%$ confidence level, and 0.32 effect size. It was planned to reach an equal number of participants in the groups. To compensate for possible problems, it was decided to collect 5\% additional data and reach a total of 105 cases.

The frequencies, ratios, mean and standard deviations of the participants in terms of different variables were presented as descriptive statistics, and numbers and \% values for categorical variables and mean and standard deviation values for continuous variables were reported in the tables. One-Way ANOVA was performed for comparisons between groups and mean \pm standard deviation values were reported. To examine whether there was a significant difference in the distribution of categorical data by the groups, Chi-square analysis was performed, and cross tables were added. In addition, the changes in the continuous variables measured in different time points were presented in line graphs and bar graphs. The significance level for all analysis results was determined as $p<0.05$. In this study, the data analysis was performed using SPPS 25 (IBM Corp. Released 2017. IBM SPSS Statistics for Windows, Version 25.0. Armonk, NY: IBM Corp) program.

\section{Results}

One hundred and twenty-eight patients were included in this study. Nineteen patients who did not meet the inclusion criteria were excluded from the study. Finally, 109 patients were randomly assigned to one of the three groups. One patient in each group was excluded due to failure to perform spinal anesthesia and one patient in the $\mathrm{PACK}+\mathrm{ACB}$ group was excluded due to surgical complications. Consequently, the data of 105 patients were analyzed (Fig. 2). Mean age and sex distribution were similar in all groups. Mean BMI and ASA classes were also comparable between the study groups. No significant difference was found between the groups about the preoperative NRS at the rest and during the movement (Table 1).

With the addition of ACB to iPACK and PAI, the 48th-h NRS score of the patients at rest and movement was significantly lower than the group to which only ACB was applied $(p<0.001)$. In addition, the 48th-h AUC movement NRS score in the iPACK + ACB group was significantly lower than in the PAI + ACB group $(p<0.05)$ (Table 2).

The NRS score of the patients in the iPACK + ACB group, which was formed at all time points at rest and movement, was significantly lower than in the ACB group $(p<0.05)$. The 6th-h rest and the 6th- and 48th-h movement NRS scores in the iPACK + ACB group were significantly lower than in the PAI + ACB group $(p<0.05)$ (Fig. 3).

At the postoperative 48 th $\mathrm{h}$, the opioid consumption of the iPACK + ACB group was lower than the ACB and PAI + ACB groups $(p<0.001)$ (Table 3$)$. The patients in the iPACK + ACB group had significantly shorter hospitalization and mobilization time than the ACB and PAI + ACB groups $(p<0.001)$. The degree of satisfaction of the patients in the $\mathrm{PACK}+\mathrm{ACB}$ and $\mathrm{PAI}+\mathrm{ACB}$ groups was significantly higher than the ACB group $(p<0.001)$ (Table 3$)$. There was no significant difference between the groups in terms of preoperative ROM and TUG values. Postoperative 2nd-day ROM values were significantly higher in the $\mathrm{iPACK}+\mathrm{ACB}$ and $\mathrm{PAI}+\mathrm{ACB}$ groups compared to the ACB group $(p<0.05)$. Postoperative 1 st- and 2 nd-day TUG test results were significantly higher in the $\mathrm{PACK}+\mathrm{ACB}$ and $\mathrm{PAI}+\mathrm{ACB}$ groups compared to the ACB group $(p<0.05)$ (Table 3).

Nausea and vomiting frequency at postoperative 4 th and 6th $\mathrm{h}$ were significantly lower in the $\mathrm{PACK}+\mathrm{ACB}$ and $\mathrm{PAI}+\mathrm{ACB}$ groups compared to the ACB group $(p<0.05)$ (Table 4). 


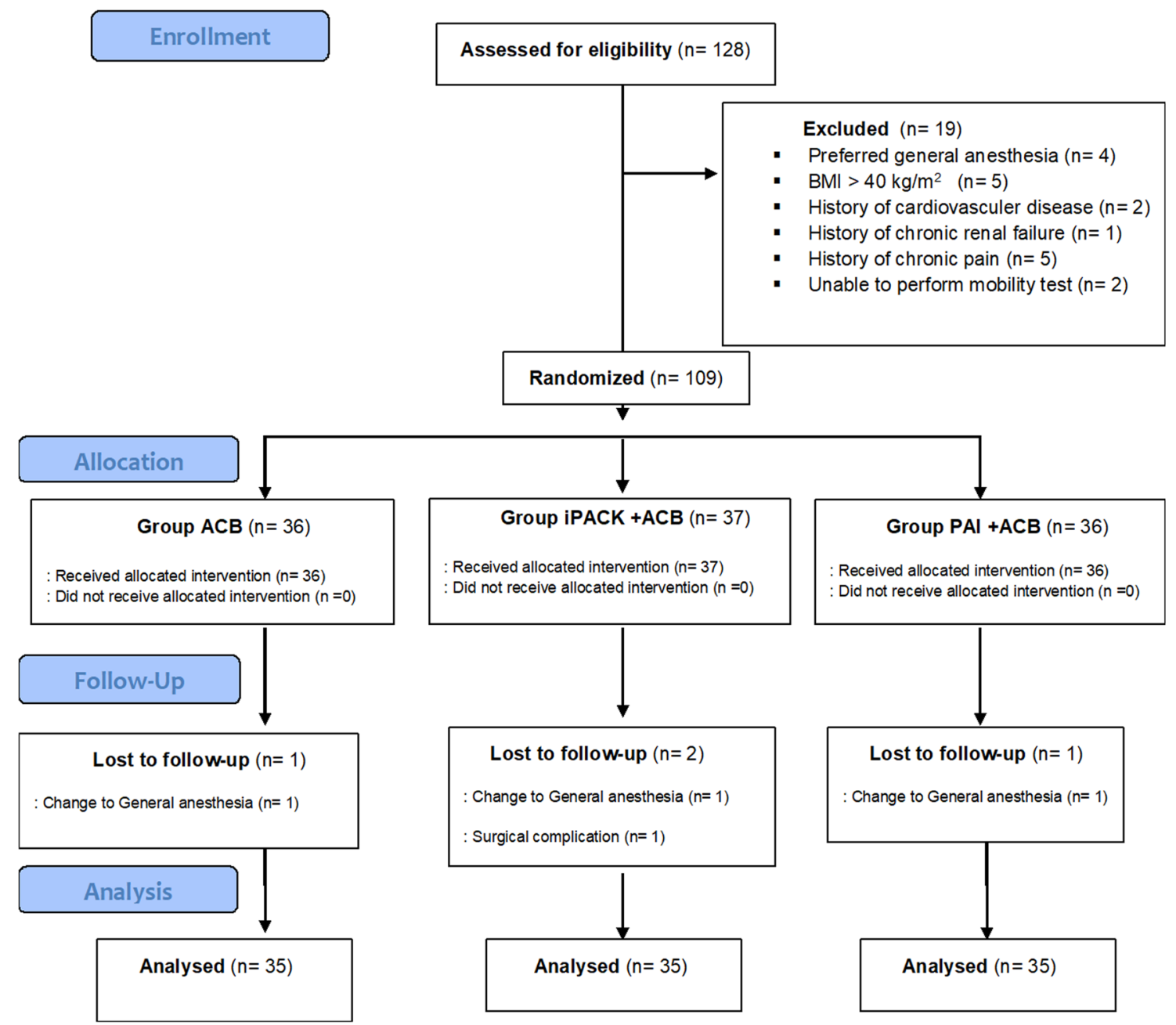

Fig. 2 Consolidated standards of reporting trials flow diagram. $B M I$ body mass index, $A C B$ adductor canal block, $i P A C K$, the interspace between the popliteal artery and capsule of the posterior knee block, respectively, PAI periarticular infiltration

\section{Discussion}

The results of this prospective, double-blind, randomized controlled trial demonstrated that ACB alone was insufficient to reduce postoperative pain in TKA, however, when it was combined with iPACK or PAI, better pain control was attained. Considering the combination of iPACK and PAI with ACB; it was shown that iPACK significantly reduced NRS and need for opioids, which occurred at the 48th hour AUC movement, compared to PAI. In addition, the combination of ACB and PACK or PAI shortened the time to hospital discharge without impairing early mobility.

Femoral and sciatic nerve blockade is accepted as the standard to provide effective analgesia after total knee arthroplasty. On the other hand, motor block that develops after femoral and sciatic blocks prevents early ambulation and reduces the time to discharge [13]. It is suggested that the analgesic effect of ACB is the result of saphenous nerve
(SN) and vastus medialis nerve (VMN) blockade [14]. VMN and $\mathrm{SN}$ have been shown to provide anteromedial innervation of the joint capsule, and the motor block is not observed after their blockade $[4,14]$. This has increased the interest in motor protection blocks such as ACB [15]. It is thought that the nerves that provide the total innervation of the knee will be blocked with the application of the block that innervates the posterior part of the joint capsule with iPACK. Adding iPACK applications to motor-protective blocks such as ACB increases the effectiveness of analgesia. A recently published study demonstrated that adding iPACK to ACB improved postoperative pain scores. However, they did not find any significant difference in opioid consumption, physical therapy performance and hospital stay [16]. In another study, iPACK + ACB reported improvement in postoperative pain scores compare to patients who underwent ACB alone. In this study, however, postoperative opioid consumption of the two group of patients were not reported [10]. Runge et al. 
Table 1 Clinicodemographic data, duration of operation and preoperative NRS at the rest and during the movement in three study groups

\begin{tabular}{lllll}
\hline & ACB $(n: 35)$ & ACB + iPACK $(n: 35)$ & ACB + PAI $(n: 35)$ & $p$ value \\
\hline $\begin{array}{l}\text { Age (years) } \\
\text { Gender }\end{array}$ & $67.1 \pm 9.0$ & $68.5 \pm 7.7$ & $71.1 \pm 6.6$ & 0.1 \\
$\quad$ Male & $16(45.7)$ & $12(34.3)$ & $14(40)$ & 0.62 \\
Female & $19(54.3)$ & $23(65.7)$ & $21(60)$ & \\
BMI $\left(\mathrm{kg} / \mathrm{m}^{2}\right)$ & $32.7 \pm 4.0$ & $33.8 \pm 3.9$ & $33.3 \pm 2.8$ & 0.45 \\
ASA & & & & 0.4 \\
II & $30(85.7)$ & $29(82.9)$ & $26(74.3)$ & \\
$\quad$ III & $5(14.3)$ & $6(17.1)$ & $9(25.7)$ & 0.77 \\
Duration of surgery (min) & $69.0 \pm 5.1$ & $69.9 \pm 5.9$ & & \\
Preoperative NRS & & & $0.4 \pm 0.7 \pm 5.7$ & 0.38 \\
At rest & $0.5 \pm 0.6$ & $0.3 \pm 0.5$ & $5.0 \pm 1.3$ & 0.53 \\
During the movement & $5.1 \pm 1.4$ & $5.4 \pm 1.2$ & & \\
\hline
\end{tabular}

Values presented as mean \pm standard deviation

ASA American Society of Anesthesiologists, $B M I$ body mass index, $A C B$ adductor canal block, $i P A C K$ the infiltration between the popliteal artery and capsule of the knee block, PAI periarticular infiltration, NRS numerical rating scale

${ }^{*} p$ value $<0.05$

\begin{tabular}{|c|c|c|c|c|}
\hline & \multicolumn{2}{|c|}{ AUC at $48 \mathrm{~h}$ for NRS (at rest) } & \multicolumn{2}{|c|}{$\begin{array}{l}\text { AUC at } 48 \mathrm{~h} \text { for NRS (during the } \\
\text { movement) }\end{array}$} \\
\hline & $(95 \% \mathrm{CI})$ & $p$ value & $(95 \% \mathrm{CI})$ & $p$ value \\
\hline $\mathrm{ACB}$ versus iPACK $+\mathrm{ACB}$ & $0.883(0.807-0.947)$ & $<0.001 *$ & $0.864(0.781-0.947)$ & $<0.001 *$ \\
\hline $\mathrm{ACB}$ versus $\mathrm{PAI}+\mathrm{ACB}$ & $0.781(0.672-0.890)$ & $0.001 *$ & $0.741(0.624-0.858)$ & $<0.001 *$ \\
\hline $\mathrm{iPACK}+\mathrm{ACB}$ versus $\mathrm{PAI}+\mathrm{ACB}$ & $0.633(0.504-0.763)$ & 0.055 & $0.660(0.532-0.788)$ & $0.022 *$ \\
\hline
\end{tabular}

Values are presented as mean, confidence interval 95\%

$A C B$ adductor canal block, $i P A C K$ the infiltration between the popliteal artery and capsule of the knee block, $P A I$ periarticular infiltration, $A U C$ area under the curve, $N R S$ numerical rating scale

${ }^{*} p$ value $<0.05$ applied similar approaches with iPACK as a popliteal plexus block (PPB), it was shown that PPB provided an effective and significant reduction in TKA postoperative pain [17]. In our study, the addition of iPACK to ACB also caused a significant decrease in pain scores at rest and movement. In addition, with the combination of these two blocks, early ambulation was achieved, and the hospital discharge time was shortened.

Another study of the clinical use of the iPACK block reported no difference in postoperative pain scores in the postoperative $12 \mathrm{~h}$ period and increase opioid consumption. On the other hand, after postoperative $36 \mathrm{~h}$, the pain scores in patients with iPACK block were lower when compare to patients with PAI block [18]. The authors concluded that iPACK block combined with ACB provides non-inferior analgesia compared to PAI when combined with ACB [16, 19]. In the present study, we found that AUC pain scores over $48 \mathrm{~h}$ in patients with iPACK block combined with ACB were lower than patients with PAI with ACB or ACB alone.
When the pain scores were evaluated at postoperative 6th $\mathrm{h}$, the iPACK + ACB group had lower pain scores. On the other hand, all three groups had higher pain scores at the postoperative 8 th $\mathrm{h}$. These higher scores were attributed to rebound pain $[15,19]$. Motor sparing blocks and multimodal analgesia enable to decrease the pain at early postoperative period in patients undergoing total knee arthroplasty. On the other hand, single-shot peripheral nerve blocks wear off at first $12 \mathrm{~h}$ after the surgery and may create rebound pain that may not treated effectively with routine postoperative pain management protocol [20]. Continuous peripheral nerve blocks is recommended to avoid the rebound pain. Nevertheless, catheter usage may create various difficulties during placement and postoperative period. Excess subcutaneous adipose tissue of the patients, strict follow-up of the catheter and confirmation of location is required. The rotational movement of the tissue around the femur may displace the catheter tip. Additional effort in placement and management of the ACB catheter may not be of significant 
Fig. 3 Results presented as mean $(95 \% \mathrm{CI})$ for NRS at rest and during the movement. $* p$ value $<0.05$ ACB between $\mathrm{iPACK}+\mathrm{ACB}, \dagger p$ value $<0.05$ $\mathrm{ACB}$ between $\mathrm{PAI}+\mathrm{ACB}, \ddagger p$ value $<0.05 \mathrm{iPACK}+\mathrm{ACB}$ between $\mathrm{PAI}+\mathrm{ACB}$. A line graph representing knee pain at rest and during the movement postoperative $48 \mathrm{~h}$ after total knee arthroplasty. $A C B$ adductor canal block, $I P A C K$ infiltration between the popliteal artery and capsule of the knee block, $P A I$ periarticular infiltration, $N R S$ numerical rating scale
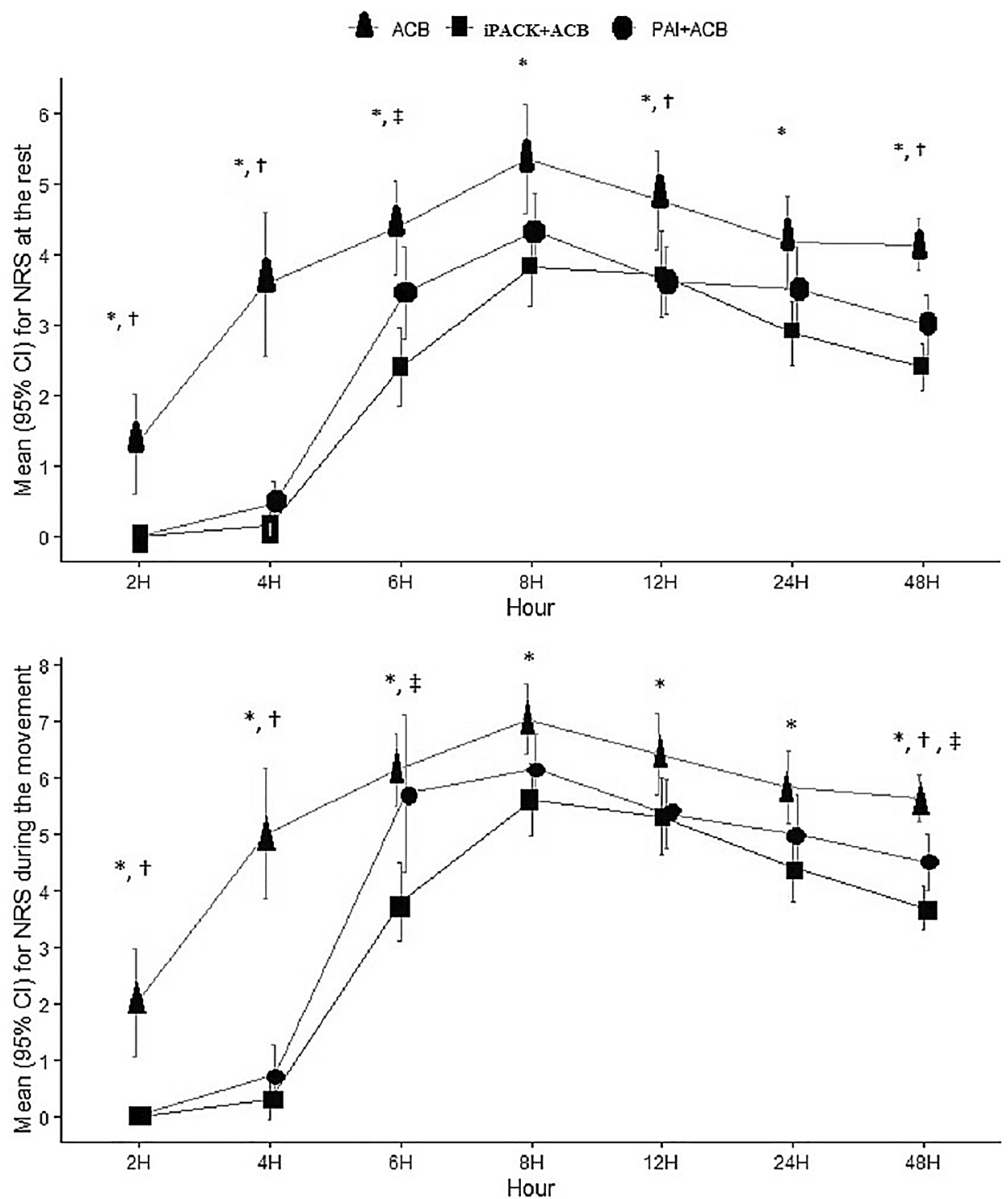

benefit for rapid mobilization of the TKA patient. In addition, combination of peripheral blocks is recommended for prevention rebound pain for patients undergoing TKA [21]. Similarly, patients in Group ACB had higher postoperative 8th $\mathrm{h}$ pain scores that were not treated effectively with routine multimodal analgesia protocol in our study. Therefore, the combination of PAI or PACK with ACB may enhance postoperative pain management.

Adding iPACK to ACB in the setting of PAI did not improve analgesia throughout the postoperative $24 \mathrm{~h}$ in patients undergoing TKA. In contrast, in the absence of PAI, the pain scores of patients with ACB + iPACK were lower at postoperative 6th, 12th, and 24th $\mathrm{h}$ [22]. This may create a condition that addition of PAI is enough when $\mathrm{ACB}$ is administered to the patients. In our study, we found a significant difference AUC result of ACB + iPACK group at 48th h seems to be better than ACB + PAI group.
However, we did not find any significant difference in postoperative function of iPACK and PAI. This may be interpreted as clinically unimportant although statistical difference between the groups. Hence, we may think that iPACK + ACB has a similar clinical outcome with $\mathrm{ACB}+\mathrm{PAI}$. However, the fact that PAI is a blind technique and administration of drugs in different combinations are seen as disadvantages. In addition, solution in the joint space may be more likely to flow out when a drainage tube is placed in the joint. Therefore, higher drug concentration may be required to reach the current drug concentration. This issue is seen as one of the main limitations of combining motor-protective blocks with PAI in terms of local anesthetic systemic toxicity (LAST) [15, 23]. In our study, we applied a total amount of $100 \mathrm{mg}$ of bupivacaine in the PAI injection and $50 \mathrm{mg}$ of bupivacaine in the ACB on guidance reduces this incidence. Therefore, 
Table 3 Analgesia outcomes, patient satisfaction, hospital stay, mobilization time, ROM and TUG in study groups

\begin{tabular}{|c|c|c|c|c|c|c|c|}
\hline & $\mathrm{ACB}$ & $\mathrm{iPACK}+\mathrm{ACB}$ & $\mathrm{PAI}+\mathrm{ACB}$ & $\begin{array}{l}p \text { value (for } \\
\text { omnibus } \\
\text { test) }\end{array}$ & $\begin{array}{l}p \text { value (ACB vs } \\
\text { iPACK }+ \text { ACB) }\end{array}$ & $\begin{array}{l}p \text { value }(\mathrm{ACB} \\
\text { vs } \mathrm{PAI}+\mathrm{ACB})\end{array}$ & $\begin{array}{l}p \text { value } \\
(\mathrm{iPACK}+\mathrm{ACB} \text { vs } \\
\mathrm{PAI}+\mathrm{ACB})\end{array}$ \\
\hline $\begin{array}{l}\text { Total tramadol }(\mathrm{mg}) \text { con- } \\
\text { sumption } 0-48 \mathrm{~h}\end{array}$ & $380 \pm 83.3$ & $197.14 \pm 61.8$ & $268.6 \pm 75.8$ & $<0.001^{*}$ & $<0.001 *$ & $<0.001^{*}$ & $<0.001 *$ \\
\hline $\begin{array}{l}\text { IV tramadol }(\mathrm{mg}) \text { con- } \\
\text { sumption (rescue analge- } \\
\text { sia, NRS }>4) 24 \mathrm{~h}\end{array}$ & $262.9 \pm 59.8$ & $160 \pm 49.7$ & $208.57 \pm 44.5$ & $<0.001^{*}$ & $<0.001^{*}$ & $<0.001 *$ & $<0.001^{*}$ \\
\hline $\begin{array}{l}\text { IV tramadol }(\mathrm{mg}) \text { con- } \\
\text { sumption (rescue analge- } \\
\text { sia, NRS }>4 \text { ) } 24-48 \mathrm{~h}\end{array}$ & $117.1 \pm 38.2$ & $37.1 \pm 49.0$ & $60.0 \pm 49.7$ & $<0.001^{*}$ & $<0.001^{*}$ & $<0.001^{*}$ & 0.136 \\
\hline $\begin{array}{l}\text { Time to first IV opioid } \\
\text { requirement (hour) }\end{array}$ & $4.5 \pm 1.6$ & $7.9 \pm 2.0$ & $7.1 \pm 1.4$ & $<0.001^{*}$ & $<0.001^{*}$ & $<0.001^{*}$ & 0.140 \\
\hline Patient satisfaction & $1.7 \pm 0.5$ & $2.9 \pm 0.4$ & $2.7 \pm 0.5$ & $<0.001^{*}$ & $<0.001^{*}$ & $<0.001^{*}$ & 0.394 \\
\hline Hospital stay (days) & $3.2 \pm 0.4$ & $2.7 \pm 0.5$ & $2.8 \pm 0.4$ & $<0.001^{*}$ & $<0.001^{*}$ & $<0.001^{*}$ & 0.498 \\
\hline Mobilization time (hours) & $7.5 \pm 1.3$ & $6.0 \pm 0.8$ & $6.8 \pm 1.0$ & $<0.001^{*}$ & $<0.001^{*}$ & $0.017^{*}$ & $0.004^{*}$ \\
\hline \multicolumn{8}{|c|}{ Range of motion (ROM) (degree) } \\
\hline Preoperative & $110.3 \pm 7.1$ & $112.1 \pm 7.6$ & $112.0 \pm 6.7$ & 0.481 & 0.552 & 0.574 & 0.996 \\
\hline Postoperative day 1 & $84.9 \pm 7.9$ & $89.1 \pm 9.5$ & $87.0 \pm 9.8$ & 0.149 & 0.125 & 0.588 & 0.588 \\
\hline Postoperative day 2 & $86.7 \pm 11.0$ & $96.6 \pm 6.9$ & $95.4 \pm 7.5$ & $<0.001^{*}$ & $<0.001^{*}$ & $0.001^{*}$ & 0.787 \\
\hline \multicolumn{8}{|l|}{ TUG (second) } \\
\hline Preoperative & $15.3 \pm 3.7$ & $15.1 \pm 4.3$ & $14.0 \pm 3.6$ & 0.571 & 0.988 & 0.784 & 0.454 \\
\hline Postoperative day 1 & $43.2 \pm 7.6$ & $38.0 \pm 7.1$ & $38.4 \pm 6.2$ & $0.004 *$ & $0.008^{*}$ & $0.014^{*}$ & 0.977 \\
\hline Postoperative day 2 & $37.9 \pm 7.8$ & $31.0 \pm 7.5$ & $31.7 \pm 6.2$ & $<0.001^{*}$ & $<0.001 *$ & $0.001^{*}$ & 0.929 \\
\hline
\end{tabular}

Numbers were presented as mean \pm standard deviation

NRS Numerical Rating Scale, $A C B$ adductor canal block, iPACK the infiltration between the popliteal artery and capsule of the knee block, $P A I$ periarticular infiltration, $R O M$ range of motion, $T U G$ time up-and-go test

$* p$ value $<0.05$

Table 4 Number of patients with nausea and/or vomiting in the study groups

\begin{tabular}{lllll}
\hline & ACB (n: 35) & iPACK + ACB (n: 35) & PAI+ ACB (n: 35) & $p$ value \\
\hline $\begin{array}{l}\text { Nausea/vomiting score } \\
\text { (none/middle/vomit- }\end{array}$ & $14 / 20 / 1$ & $27 / 7 / 1$ & $20 / 11 / 4$ & $0.007^{*}$ \\
ing) $4 \mathrm{~h}$ & & & \\
$\begin{array}{c}\text { Nausea/vomiting score } \\
\text { (none/middle/vomit- } \\
\text { ing) } 6 \mathrm{~h}\end{array}$ & $20 / 15 / 0$ & $29 / 5 / 1$ & $30 / 3 / 2$ & $0.005^{*}$ \\
\hline
\end{tabular}

$A C B$ adductor canal block, $i P A C K$ the infiltration between the popliteal artery and capsule of the knee block, $P A I$ periarticular infiltration;

$* p$ value $<0.05$
iPACK block application may be preferred to avoid possible complications.

The postoperative analgesia protocol for TKA has been progressively improved. It includes a combination of epidural, intrathecal morphine, a single-shot femoral nerve block or ACB; ACB combined with local infiltration anesthesia, and finally, local infiltration anesthesia combined with continuous ACB (CABC) [24, 25]. A perioperative multimodal regimen is applied to the patients with spinal anesthesia performed with a short-acting local anesthetic. In this way, the blockage caused by postoperative spinal anesthesia is quickly eliminated, the peripheral nerve block added and the analgesic agents, which are the other components of multimodal anesthesia, provide postoperative analgesia allowing for early mobilization [25]. Thus, effective analgesia in the early postoperative period may provide to patient to exercise and early improvement in mobility, thereby decrease length of stay by facilitating functional recovery. In our study, we assessed early postoperative recovery with ROM and TUG values and found a significant difference in these values in $\mathrm{PACK}+\mathrm{ACB}$ and $\mathrm{PAI}+\mathrm{ACB}$ group compared to ACB. However, we did not find any 
significant difference in postoperative function of iPACK and PAI. When iPACK or PAI were added to the ACB, the postoperative rehabilitation program produced better results in terms of 2nd postoperative day ROM values compared to those of the patients who received only ACB.

In tibial nerve block (TNB) or iPACK block application, there is no difference in cumulative morphine consumption up to $48 \mathrm{~h}$, rescue analgesia time, and functional results such as knee range of motion and TUG test in the first 3 days after surgery. The hospital stay was also shorter in the iPACK group than in the TNB group. Considering early mobilization and earlier discharge, iPACK block may be a preferred motor-sparing alternative to TNB with a lower incidence of foot drop and an increased likelihood. Drop foot was observed in 2 of 411 patients who underwent iPACK block $(0.48 \%)$ [11]. We did not observe any foot drop with iPACK in our trial. In addition, better results were obtained in the iPACK and PAI group with added ACB in terms of ambulation time, discharge, and patient general satisfaction. As a result, a significant reduction in hospital stay is achieved. Concomitantly, the patient's degree of satisfaction with the surgical procedure increases.

A meta-analysis has found that periarticularly administered morphine has no significant effect on the 48-h pain score at rest and in motion but reduces postoperative opioid consumption [26]. Nevertheless, it has been shown that analgesia provided through peripheral opioid receptors by adding opioids to solutions using PAI is prolonged up to $48 \mathrm{~h}$. This reduces the incidence of side effects from opioid administration. After TKA surgery, the patients' analgesic demand may be increased by blocking between the endogenous opioid and opioid receptor interaction. Intraarticular opioid application may alleviate this nociceptive effects of inflammation mainly by binding peripheral opioid receptors. It has been suggested that systemic administration of opioids also produce large proportion of its acute analgesic effect by binding on peripheral opioid receptors. Hence, periarticular local anesthetic cocktails including morphine may increase analgesic efficacy of opioids by avoiding opioid related systemic side effects $[27,28]$. We use the combination of morphine and local anesthetic without adding steroid or nonsteroidal anti-inflammatory agent in periarticular injection cocktail with intravenous dexamethasone to prolong analgesic effect of local anesthetics with assumption of reduction in opioid related side effects that may be seen after intravenous consumption [29].

A meta-analysis has found that iPACK has moderate evidence-related analgesic outcome of iPACK block and this was not correlated with functional outcomes. There is limited evidence to support the use of iPACK as a supplement to ACB for postoperative 12th-h pain after total knee arthroplasty. However, in the meta-analysis, the postoperative pain assessment was done in a 12-h time interval [30].
In addition, this meta-analysis has heterogeneity in the study group, and the evaluation of different methodology in studies which were evaluated. In our study, we choose to evaluate 48 -h pain scores, since rehabilitation application, which is started at the postoperative 48th $\mathrm{h}$ in patients who have undergone TKA, has a positive effect on the clinical results [31]. Therefore, our study, which includes these three different block combinations, has more clinical significance than other similar studies since it evaluates total pain in the first $48 \mathrm{~h}$ postoperatively.

Our study has several limitations. We applied single-shot ACB in all patients and continuous manner with use of catheter may yield better results in terms of opioid consumption of the patients and provide earlier mobilization for patients. There is for future studies consider the comparison of iPACK and PAI added to the continuous ACB. Our second limitation is the use of tramadol in the postoperative multimodal analgesia management of the patients. This study was conducted during COVID-19 pandemic and shortage of intravenous opioid was seen due to overwhelming use in the management of COVID-19 patients in the intensive care unit [32]. Studies with multimodal analgesia protocols using strong opioids are needed. Another limitation is the use of unequal amounts of local anesthetics between the groups. The dose used in the PAI group was higher than that in the iPACK block groups, although similar to those in previous studies [25]. However, the minimum effective concentration and volume of local anesthetic have not yet been determined for these blocks.

In conclusion, in the present study, the addition of iPACK block to multimodal analgesia regimen with ACB improved postoperative 48-h AUC pain scores, decrease opioid consumption compare to addition of PAI. In addition, it reduces the time to patient mobilization and hospital discharge. Therefore, the addition of iPACK block to multimodal analgesia regimen including ACB can be offered for patients undergoing TKA. Further studies examining the long-term functional and analgesic effect of iPACK + ACB (single shot vs continuous infusion), dose and concentration of local anesthetics are needed.

Acknowledgements The authors acknowledge physiotherapists, nurses, and anesthetists involved in the trial at Karaman Training and Research Hospital for their obliging and invaluable support. This work was guaranteed by Karaman Training and Research Hospital as declared in the clinical trial registration.

Author contributions Study design/planning: TE and BB. Data analysis and interpretation: TE. Drafting and writing the paper: all the authors. Revision and approval of the final manuscript: all the authors.

\section{Declarations}

Conflict of interest The authors declare no conflict of interest. 


\section{References}

1. Kopp SL, Børglum J, Buvanendran A, Horlocker TT, Ilfeld BM, Memtsoudis SG, Neal JM, Rawal N, Wegener JT. Anesthesia and analgesia practice pathway options for total knee arthroplasty: an evidence-based review by the American and European societies of regional anesthesia and pain medicine. Reg Anesth Pain Med. 2017;42:683-97.

2. Elmallah RK, Chughtai M, Khlopas A, Newman JM, Stearns KL, Roche M, Kelly MA, Harwin SF, Mont MA. Pain control in total knee arthroplasty. J Knee Surg. 2018;31:504-13.

3. Kehlet $\mathrm{H}$, Andersen L $\emptyset$. Local infiltration analgesia in joint replacement: the evidence and recommendations for clinical practice. Acta Anaesthesiol Scand. 2011;55:778-84.

4. Kuang MJ, Ma JX, Fu L, He WW, Zhao J, Ma XL. Is adductor canal block better than femoral nerve block in primary total knee arthroplasty? A GRADE analysis of the evidence through a systematic review and meta-analysis. J Arthroplasty. 2017;32:3238-48.

5. Abdallah FW, Madjdpour C, Brull R. Is sciatic nerve block advantageous when combined with femoral nerve block for postoperative analgesia following total knee arthroplasty? A meta-analysis. Can J Anaesth. 2016;63:552-68.

6. Niesen AD, Harris DJ, Johnson CS, Stoike DE, Smith HM, Jacob AK, Amundson AW, Pawlina W, Martin DP. Interspace between popliteal artery and posterior capsule of the knee (IPACK) injectate spread: a cadaver study. J Ultrasound Med. 2019;38:741-5.

7. Tran J, Arango LG, Peng P, Sinha SK, Agur A, Chan V. Evaluation of the iPACK block injectate spread: a cadaveric study. Reg Anesth Pain Med. 2019;208:100355.

8. Kandarian B, Indelli PF, Sinha S, Hunter OO, Wang RR, Kim E, Kou A, Mariano ER. Implementation of the IPACK (infiltration between the popliteal artery and capsule of the knee) block into a multimodal analgesic pathway for total knee replacement. Korean J Anesthesiol. 2019;72:238-44.

9. Thobhani S, Scalercio L, Elliott CE, Nossaman BD, Thomas LC, Yuratich D, Blan K, Osteen K, Patterson ME. Novel regional techniques for total knee arthroplasty promote reduced hospital length of stay: an analysis of 106 patients. Ochsner J. 2017;17:233-8.

10. Sankineani SR, Reddy ARC, Eachempati KK, Jangale A, Reddy AVG. Comparison of adductor canal block and IPACK block (interspace between the popliteal artery and the capsule of the posterior knee) with adductor canal block alone after total knee arthroplasty: a prospective control trial on pain and knee function in immediate postoperative period. Eur J Orthop Surg Traumatol. 2018;28:1391-5.

11. Chan E, Howle R, Onwochei D, Desai N. Infiltration between the popliteal artery and the capsule of the knee (IPACK) block in knee surgery: a narrative review. Reg Anesth Pain Med. 2021;46:784-805.

12. Kim DH, Beathe JC, Lin Y, YaDeau JT, Maalouf DB, Goytizolon E, Garnet C, Ranawat AS, Su EP, Mayman DJ, Memtsoudis SG. Addition of infiltration between the popliteal artery and the capsule of the posterior knee and adductor canal block to periarticular injection enhances postoperative pain control in total knee arthroplasty: a randomized controlled trial. Anesth Analg. 2019;129:526-35.

13. Zhang Z, Yang Q, Xin W, Yixuan Z. Comparison of local infiltration analgesia and sciatic nerve block as an adjunct to femoral nerve block for pain control after total knee arthroplasty: a systematic review and meta-analysis. Medicine (Baltimore). 2017;96:6829.

14. Laurant DB, Peng P, Arango LG, Niazi AU, Chan VW, Agur A, Perlas A. The nerves of the adductor canal and the innervation of the knee: an anatomic study. Reg Anesth Pain Med. 2016;41:321-7.

15. Sawhney M, Mehdian H, Kashin B, Ip G, Bent M, Joyce C, McPherson M, Bowry R. Pain after unilateral total knee arthroplasty: a prospective randomized controlled trial examining the analgesic effectiveness of a combined adductor canal peripheral nerve block with periarticular infiltration versus adductor canal nerve block alone versus periarticular infiltration alone. Anesth Analg. 2016;122:2040-6.

16. Patterson ME, Vitter Y, Bland K, Nossaman BDMD, Thomas LC, Chimento GF. The effect of the IPACK block on pain after primary TKA: a double-blinded, prospective. Randomized Trial J Arthroplasty. 2020;35:173-7.

17. Runge C, Bjørn S, Jensen JM, Nielsen ND, Vase M, Holm C, Bendtsen TF (2018) The analgesic effect of a popliteal plexus blockade after total knee arthroplasty: a feasibility study. Acta Anaesthesiol Scand 24

18. Kertkiatkachorn W, Kampitak W, Tanavalee A, Ngarmukos S. Adductor canal block combined with iPACK (interspace between the popliteal artery and the capsule of the posterior knee) block vs periarticular injection for analgesia after total knee arthroplasty: a randomized noninferiority trial. J Arthroplasty. 2021;36:122-9.

19. Muñoz-Leyva F, Cubillos J, Chin KJ. Managing rebound pain after regional anesthesia. Korean J Anesthesiol. 2020;73:372-83.

20. Woo JH, Lee HJ, Oh HW, Lee JW, Baik HJ, Kim YJ. Perineural dexamethasone reduces rebound pain after ropivacaine single injection interscalene block for arthroscopic shoulder surgery: a randomized controlled trial. Reg Anesth Pain Med. 2021;46:965-70.

21. Youm YS, Cho SD, Cho HY, Hwang CH, Jung SH, Kim KH. Preemptive femoral nerve block could reduce the rebound pain after periarticular injection in total knee arthroplasty. J Arthroplasty. 2016;31:1722-6.

22. Hussain N, Brull R, Sheehy B, Dasu M, Weaver T, Abdallah FW. Does the addition of iPACK to adductor canal block in the presence or absence of periarticular local anesthetic infiltration improve analgesic and functional outcomes following total knee arthroplasty? A systematic review and meta-analysis. Reg Anesth Pain Med. 2021;46:713-21.

23. Ross JA, Greenwood AC, Sasser P, Jiranek WA. Periarticular injections in knee and hip arthroplasty: where and what to inject. J Arthroplast. 2017;32:77-80.

24. Kampitak W, Tanavalee A, Ngarmukos S, Amarase C, Apihansakorn R, Vorapalux P. Does adductor canal block have a synergistic effect with local infiltration analgesia for enhancing ambulation and improving analgesia after total knee arthroplasty? Knee Surg Relat Res. 2018;30:133-41.

25. Kampitak W, Tanavalee A, Ngarmukos S, Amarase C. Opioidsparing analgesia and enhanced recovery after total knee arthroplasty using combined triple nerve blocks with local infiltration analgesia. J Arthroplasty. 2019;34:295-302.

26. Zhang Y, Mi F, Zhao H, Xie D, Shi X. Effect of morphine added to multimodal cocktail on infiltration analgesia in total knee arthroplasty A meta-analysis of randomized controlled trials. Medicine (Baltimore). 2019;98:17503.

27. Wang Q, Sun J, Hu Y, Zeng Y, Hu J, Yang J, Kang P. Effects of morphine on peri-articular infiltration analgesia in total knee arthroplasty: a prospective, double-blind, randomized controlled trial. Int Orthop. 2020;44:2587-95.

28. Stein C. Opioid analgesia: recent developments. Curr Opin Support Palliat Care. 2020;14:112-7.

29. Xu H, Zhang S, Xie J, Lei Y, Cao G, Pei F. Multiple doses of perioperative dexamethasone further improve clinical outcomes after total knee arthroplasty: a prospective, randomized controlled study. J Arthroplasty. 2018;33:3448-54. 
30. Albrecht E, Wegrzyn J, Dabetic A, El-Boghdadly K. The analgesic efficacy of iPACK after knee surgery: a systematic review and meta-analysis with trial sequential analysis. J Clin Anesth. 2021;72:110305.

31. Perlas A, Kirkham KR, Billing TC, Brull R, Gandhi R, Chan VW. The impact of analgesic modality on early ambulation following total knee arthroplasty. Anesth Pain Med. 2013;38:334-9.

32. DeMik DE, Carender CN, Shamrock AG, Callghan JJ, Bedard NA. Opioid use after total knee arthroplasty: does tramadol have lower risk than traditional opioids? J Arthroplasty. 2020;35:1558-62.
Publisher's Note Springer Nature remains neutral with regard to jurisdictional claims in published maps and institutional affiliations. 